\title{
Instantaneous local wave vector estimation from multi-spacecraft measurements using few spatial points
}

\author{
T. D. Carozzi, A. M. Buckley, and M. P. Gough \\ Space Science Centre, University of Sussex, Brighton, England \\ Received: 30 September 2003 - Revised: 14 March 2004 - Accepted: 14 April 2004 - Published: 14 July 2004 \\ Part of Special Issue "Spatio-temporal analysis and multipoint measurements in space"
}

\begin{abstract}
We introduce a technique to determine instantaneous local properties of waves based on discrete-time sampled, real-valued measurements from 4 or more spatial points. The technique is a generalisation to the spatial domain of the notion of instantaneous frequency used in signal processing. The quantities derived by our technique are closely related to those used in geometrical optics, namely the local wave vector and instantaneous phase velocity. Thus, this experimental technique complements ray-tracing. We provide example applications of the technique to electric field and potential data from the EFW instrument on Cluster. Cluster is the first space mission for which direct determination of the full 3-dimensional local wave vector is possible, as described here.
\end{abstract}

Key words. Electromagnetics (wave propagation) - Space plasma physics (experimental and mathematical techniques) - General or miscellaneous (techniques applicable in three or more fields)

\section{Introduction}

There are many general multipoint spatio-temporal algorithms in use in experimental space physics for the study of waves and structures. A survey of some of these techniques is given in the analysis methods book (Paschmann and Daly, 1998). There are time domain-based methods, like the discontinuity analyser which measures structures using timing differences between measurement points. Then there are frequency domain-based methods such as two point interferometry proposed in Kintner et al. (1984). There are techniques which take advantage of the vectorial or polarimetric aspects of waves (Storey and Lefeuvre, 1974), such as the $k$-filtering (Pinçon and Motschmann, 1998) or wave telescope technique (Glassmeier et al., 2001). Basically all the techniques to date for measuring wave properties are based on two fun-

Correspondence to: T. D. Carozzi

(t.carozzi@sussex.ac.uk) damental assumptions, namely stationarity and homogeneity. Unfortunately, in space physics most phenomenon are both nonstationary and inhomogeneous. Despite some recent techniques based on wavelets (Balikhin et al., 2003) few multipoint techniques are available in these cases. In what follows we will present a novel analysis method which is applicable to non-stationary and inhomogeneous wave phenomena. This is accomplished by basing the analysis on the instantaneous and local properties of the observed field. The method is based on the concepts of instantaneous frequency (Cohen, 1995) and local wave number (Beall et al., 1982) and is implemented via the Hilbert transform. We present the method from the perspective of multipoint measurements in space physics but the treatment can easily be generalised to other subjects areas.

\section{Continuum theory of the instantaneous frequency and local wave vector}

The method presented in this paper is a multivariate, multidimensional generalisation of the instantaneous frequency (IF), (see Cohen, 1995; Boashash, 1992), extended to include the three-dimensional spatial domain. In the construction of a spatial domain analogue of the IF, we rely heavily on the concept of the local wave vector from geometrical optics (Whitham, 1974). We first present the necessary background and concepts in terms of a continuum field before dealing in the next section with the discrete nature of actual multipoint measurements.

Consider a physical quantity defined on a time-space continuum, $(t, \mathbf{x})$, which exhibits wave-like phenomena, fluctuations, or structures. Although physically direct measurements of the quantity are normally real valued, it is fruitful to consider the measurement of the physical quantity $s_{r}$ as the real part of a complex field $z$. Explicitly, we model the physical field as the sum of the measured real part $s_{r}$ and an imaginary part $s_{i}$, such that

$z(t, \mathbf{x})=s_{r}(t, \mathbf{x})+\mathrm{i} s_{i}(t, \mathbf{x})$. 


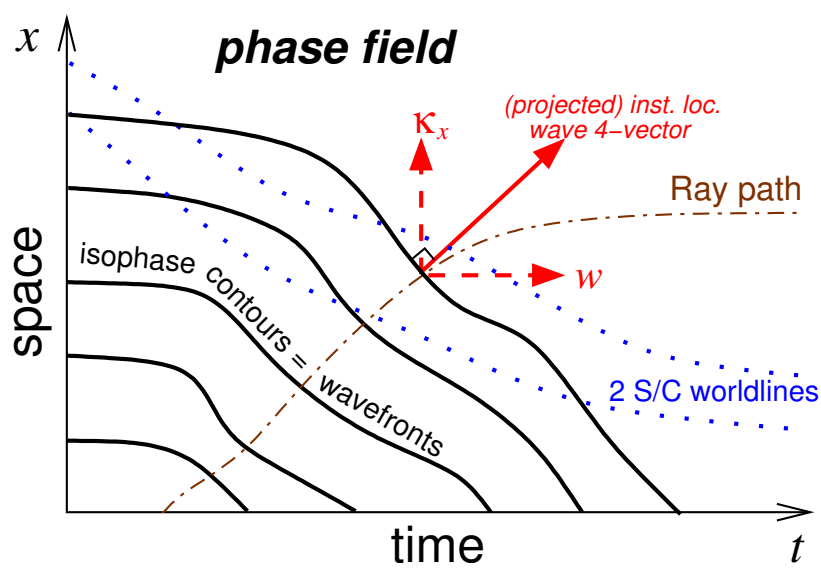

Fig. 1. Illustration of a 2-dimensional projection (time and one spatial component) of the instantaneous local wave 4-vector under the assumptions of geometrical optics and isotropy. From the phase field of a physical quantity one can define wavefronts in this illustration as isophase contour lines (solid curves) and a ray path (dashed curve) that are everywhere orthogonal to the wavefronts. The instantaneous local wave vector $\boldsymbol{\kappa}$ is the spatial gradient of the phase field and, in the geometrical optics approximation, $\boldsymbol{\kappa}$ is tangent to the ray path. The problem addressed in this paper is how to estimate $\boldsymbol{\kappa}$ from real valued discrete measurements taken along world lines (dotted curves).

Surprisingly, although we have not directly measured the imaginary part, one can determine it from the real part as long as the field is analytically well behaved. An example of this is the Kramers-Kronig relation, which in fact originally related the imaginary part of the dispersion index to the real part by assuming causality. Mathematically, the imaginary part is related to the real part through the Hilbert transform (HT), that is the HT of a real function is its imaginary part and the inverse HT of the imaginary part of a function gives its real part. Thus, by using the HT we can construct the analytic field, such as $z$, from a real function $s_{r}$.

Once we are in possession of an analytical field it is straightforward to define the amplitude $A$

$A=|z|=\sqrt{s_{r}^{2}+s_{i}^{2}}$

and the phase $\phi$

$\phi=\arg z=\arctan s_{r} / s_{i}$

of the field so that we can write the field in the polar form

$z(t, \mathbf{x})=A(t, \mathbf{x}) e^{\mathrm{i} \phi(t, \mathbf{x})}$.

Note that both $\phi$ and $A$ are real valued fields on the spacetime continuum.

The propagation of a wave mode $m$ is ultimately determined by the local refractive index $n_{m}(\mathbf{x})$ which depends on the local properties of the medium and characteristics of the mode. Unfortunately, one does not always have direct measurements of $n_{m}(\mathbf{x})$, but under the simplifying approximation that the medium varies slowly over a wavelength, known as the geometrical optics approximation, one can show (Born and Wolf, 1999) that it is given by

$|n(t, \mathbf{x})|^{2}=|\nabla \phi(t, \mathbf{x})|^{2}$.

This equation is known as the eikonal equation and it implies that the dynamics of wave propagation is determined through the dynamics of the phase. Surfaces with constant phase, $\phi(\mathbf{x})=$ const, are called wave fronts, whereas characteristics, or ray paths, are the curves which are everywhere orthogonal to the wave fronts. Thus, in geometrical optics, the rays follow the wave front normals $\nabla \phi$. In particular, the quantity

$\kappa(t, \mathbf{x}):=-\nabla \phi$,

is known as the local wave vector. These concepts are illustrated in Fig. 1.

The definition of the instantaneous local wave vector $\boldsymbol{\kappa}$ is the spatial compliment of the instantaneous local angular frequency, $w$, defined as the temporal gradient of the wave phase or

$w(t, \mathbf{x}):=\frac{\partial \phi}{\partial t}$.

The name for $w$ is motivated from the fact that it is a generalisation of frequency defined at instant $t$ and local spatial point $\mathbf{x}$. It can be shown that the temporal average of the instantaneous frequency is equal to the spectral average of the frequency spectrum, that is, under appropriate normalisation,

$\langle w\rangle=\int w(t)|z(t)|^{2} \mathrm{~d} t=\int \omega|Z(\omega)|^{2} \mathrm{~d} \omega=\langle\omega\rangle$,

where $Z(\omega)$ is the Fourier transform of $z(t)$ (see Cohen, 1995).

In the above we are assuming that there is only one wave. By this we mean that the amplitude and phase of the signal in Eq. (1) corresponds to a unique wave as opposed to a superposition of independent waves such as

$z=A_{1} e^{\mathrm{i} \phi_{1}}+A_{2} e^{\mathrm{i} \phi_{2}}+\ldots$

The former case is known as a single component wave. In the current paper we will only consider single component waves, although we developed an extension of our analysis to treat multiple component waves.

To interpret $w$ and $\boldsymbol{\kappa}$, assume that the phase is slowly varying around some point in space-time $\left(t_{0}, \mathbf{x}_{0}\right)$ and perform a multivariate Taylor expansion with the result

$\phi(t, \mathbf{x})=\phi\left(t_{0}, \mathbf{x}_{0}\right)+\left(t-t_{0}\right) \frac{\partial \phi}{\partial t}+\left(\mathbf{x}-\mathbf{x}_{0}\right) \nabla \phi+\ldots$

so that close to any arbitrary point $\left(t_{0}, \mathbf{x}_{0}\right)$ the field of the physical quantity can be approximated by

$z(t, \mathbf{x}) \approx A e^{\mathrm{i} \phi_{0}} \exp (\mathrm{i}(w t-\boldsymbol{\kappa} \cdot \mathbf{x}))$

with

$\phi_{0}:=\phi\left(t_{0}, \mathbf{x}_{0}\right)-w t_{0}+\boldsymbol{\kappa} \cdot \mathbf{x}_{0}$, 
where we have used the definitions (6) and (5). Clearly this shows that for a slowly varying field, the field can be approximated by a locally plane wave and that $w$ and $\boldsymbol{\kappa}$ correspond to the local plane wave frequency and wave vector, respectively. Taken together, $(w, \boldsymbol{\kappa})$, the instantaneous local frequency and wave vector constitute a wave 4 -vector.

Finally, the ratio of $w$ and $\kappa$

$v_{p}=\frac{w}{|\kappa|}$

is the instantaneous local phase velocity of the wave.

It should be stressed that the above discussion on ray paths assumes that the medium is isotropic. In a general anisotropic medium, such as the magnetoactive plasma of the Earth's magnetosphere, $\boldsymbol{\kappa}$ is not usually parallel to the path of the ray.

\section{Estimating the instantaneous local frequency and wave vector}

Having introduced the concept of the instantaneous local frequency $w$ and the instantaneous local wave vector $\boldsymbol{\kappa}$, we now turn to the problem of obtaining numerical estimates of these quantities from actual measurements along multiple world lines; that is, given real measurements of a physical field taken along at least 4 known space-time trajectories, we wish to find an algorithm which estimates $(w, \boldsymbol{\kappa})$.

To simplify the discussion we specialise and imagine 4 identical spacecraft $(\mathrm{s} / \mathrm{c})$ moving along simple trajectories or world lines, by which we mean that the world lines and their derivatives are continuous and that the positions of the spacecraft relative to each other do not change quickly with respect to frequencies of the waves we are interested in. Along these world lines, the spacecraft sample a real scalar field at regular discrete time intervals. We index the spacecraft simply as $\alpha=1,2,3,4$ and their trajectories are given as

$\mathbf{r}^{(\alpha)}(\sigma)=\left(t^{(\alpha)}(\sigma), \mathbf{x}^{(\alpha)}(\sigma)\right)$

in a common reference system and where $\sigma$ is the serial data sample number since the instrument started measuring; that is the first sample is $\sigma=1$, the second $\sigma=2$, and so on. Thus, for any given sample number $\sigma$, we know the absolute positions and time of all the spacecraft relative to a common coordinate system. We assume that the sampled data has been processed in such a way that

$t^{(1)}(\sigma)=t^{(2)}(\sigma)=t^{(3)}(\sigma)=t^{(4)}(\sigma)$,

at least for the samples of interest $\sigma$. This can be achieved using a multiple timelines joining algorithm (Paschmann and Daly, 1998). We only treat the general case where $\mathbf{x}^{(\alpha)}(\sigma)$ do not all lie in the same plane for any fixed $\sigma$. Finally, we will assume that all the measurements are noise free.

As was stressed in the previous section, the ILW analysis relies on the complex valued analytical field even though the data taken by the spacecraft is real valued. The first problem is therefore that we need to estimate the imaginary part of the analytical field based on the real measurements. The solution to this problem has been given elsewhere, see Cohen (1995) and Boashash (1992). Gabor suggested a unique method to achieve this based on the Hilbert transform. The analytic field in the continuum non-discrete case is constructed as

$z^{(\alpha)}\left(t, \mathbf{x}^{(\alpha)}(t)\right)=s_{r}^{(\alpha)}\left(t, \mathbf{x}^{(\alpha)}(t)\right)+s_{i}^{(\alpha)}\left(t, \mathbf{x}^{(\alpha)}(t)\right)$,

where

$s_{i}:=H_{t}\left(s_{r}\right)=\mathcal{P} \int \frac{s_{r}\left(t^{\prime}\right)}{t-t^{\prime}} \mathrm{d} t^{\prime}$

is the HT, where $\mathcal{P}$ denotes the Cauchy principle value of the integral.

There are many numerical methods to estimate the HT (Hahn, 1997) and construct the analytical signal, but we will only describe the basic idea which applies to discretely sampled, nonreal-time data. The analytical signal is computed as follows: first select the interval of interest for the real measured discrete time series $s_{r}^{(\alpha)}(\sigma)$ of length $N$ samples; then Fourier transform this series and discard all the negative frequencies, that is, all frequencies above the Nyquist frequency; and then inverse Fourier transform the result back into the time domain. The analytical signal estimate can therefore be written

$$
\S^{(\alpha)}(\sigma)=\frac{1}{N} \sum_{f=0}^{(N-1) / 2} \sum_{\sigma^{\prime}=0}^{N-1} s_{r}^{(\alpha)}\left(\sigma^{\prime}\right) \exp \left(\mathrm{i} 2 \pi \frac{f\left(\sigma-\sigma^{\prime}\right)}{N}\right)
$$

where we note that the first summation goes only over the first half of the frequency indices or positive frequencies, and excludes the second half of the frequency indices or negative frequencies. The exclusion of these negative frequencies which are complex conjugate to the positive frequencies means that the analytical signals $s^{(\alpha)}(\sigma)$ are in general complex, as expected.

Note that the analytic field estimated according to Eq. (10) is based entirely in the time domain and one may wonder how this relates to a truly multi-dimensional analytic signal such as defined by Hahn (1997). With only the minimal number of spatial point measurements considered here, it is not possible to Hilbert transform along the spatial dimension. But, for the application to wave analysis considered here, a more appropriate notion of the multi-dimensional analytic signal is given by Perrin et al. (1986). Fortunately, this latter notion is entirely consistent with the construction of the multidimensional analytical field given here, namely, that the analytic signal is based on the Hilbert transform in the time domain only and not in the spatial domains. Explicity we use Perrin's notion of the analytic signal with the reasonable criteria that the wave vector components can be both positive or negative but that the frequency must be positive.

From the 4 analytic signals $z^{(\alpha)}(\sigma)$, it is straightforward to compute the amplitude and phase for each sample from each spacecraft, denoted $A^{(\alpha)}(\sigma)$ and $\phi^{(\alpha)}(\sigma)$, respectively. The only problem is how to choose the appropriate $2 \pi$ branch for a phase value since the arctan function is multi-valued. 
Fortunately we are not interested in the absolute phase but only its spatial and temporal gradients and so we only need the phase difference between adjacent samples; even so there is no foolproof method to do this. The basic algorithm is to calculate all phase differences modulo $2 \pi$, (the argument of the field can be taken to be the principle branch of Eq. (3)). This assumes that $2 \pi / \kappa$ is not less than the minimum interspacecraft distance.

However, this fundamental wave number may not be the true wave number because the fundamental is indistinguishable from any integer multiple of the fundamental. This phenomenon is known as spatial-aliasing and can only be entirely removed if the wave can be filtered by wavelength, which unfortunately is seldom possible in space physics instrumentation for long wavelengths. The analogous problem with $w$ does not exist in practice since modern digital instruments usually have appropriate temporal anti-alias filters.

In order to estimate $w$, we must discretise the continuous definition (6) and so we propose

$$
\begin{aligned}
& w^{(\alpha)}(\sigma+1 / 2)=\frac{\left[\Delta_{t} \phi^{(\alpha)}\right]_{\bmod \pm \pi}}{\Delta t} \\
& =\frac{\left[\phi^{(\alpha)}(\sigma+1)-\phi^{(\alpha)}(\sigma)\right]_{\bmod \pm \pi}}{t(\sigma+1)-t(\sigma),}
\end{aligned}
$$

where we have used the symmetric modulus operation ${ }^{1}$, similar to Bülow and Sommer (2001), defined as

$[\varphi]_{\bmod \pm \pi}:=\bmod (\varphi+\pi, 2 \pi)-\pi$.

The instantaneous local wave vector is, according to Eq. (5), the spatial gradient of the phase but since it is a multidimensional derivative it is slightly more complicated to estimate than $w$. Techniques to calculate spatial gradients from multipoint measurements is given in Paschmann and Daly (1998). We estimate $\boldsymbol{\kappa}$ from a 4-point spatial gradient for sample $\sigma$ by using

$\boldsymbol{\kappa}(\sigma)=-\left(\stackrel{(\alpha, 1)}{\Delta_{\mathbf{X}}} \mathbf{x}^{(\alpha)}(\sigma)\right)^{-1}\left[\stackrel{(\alpha, 1)}{\Delta_{\mathbf{x}}} \phi^{(\alpha)}(\sigma)\right]_{\bmod \pm \pi}$

which in component form is

$\boldsymbol{\kappa}=\left(\begin{array}{c}\kappa_{x} \\ \kappa_{y} \\ \kappa_{z}\end{array}\right)$,

$\stackrel{(\alpha, 1)}{\Delta_{\mathbf{x}}} \mathbf{x}^{(\alpha)}:=\left(\begin{array}{ll}x^{(2)}-x^{(1)} & y^{(2)}-y^{(1)} z^{(2)}-z^{(1)} \\ x^{(3)}-x^{(1)} & y^{(3)}-y^{(1)} z^{(3)}-z^{(1)} \\ x^{(4)}-x^{(1)} y^{(4)}-y^{(1)} z^{(4)}-z^{(1)}\end{array}\right)$,

$\stackrel{(\alpha, 1)}{\Delta_{\mathbf{x}}} \phi^{(\alpha)}:=\left(\begin{array}{l}\phi^{(2)}-\phi^{(1)} \\ \phi^{(3)}-\phi^{(1)} \\ \phi^{(4)}-\phi^{(1)}\end{array}\right)$.

Note that here, the 4-point spatial gradient operator $\stackrel{(\alpha, 1)}{\Delta}$ is calculated using differences with respect to spacecraft number 1 but could easily be reformulated so as to be computed with respect to any of the spacecraft. Also note that if the original measurements $s_{r}^{(\alpha)}(\sigma)$ have been temporally antialiased filtered, then the modular division in Eq. (11) is not necessary. However, since it is not usually feasible to spatially anti-alias filter data from spacecraft missions, the $\boldsymbol{\kappa}$ estimate is susceptible to spatially aliased signals, that is, without additional knowledge, the $\boldsymbol{\kappa}$ estimate (12) as it stands selects the largest wavelengths components consist with the measured phase differences. Thus, the smallest detectable wavelength component will always be larger than half the minimum inter-spacecraft separation. Furthermore, we cannot distinguish between wave number components which are congruent modulo $2 \pi / \Delta x$.

\section{Instantaneous local spatio-temporal amplitude mod- ulation and bandwidth}

Up until now we have only discussed the oscillatory aspects of fields which are fully determined by the phase. Now we turn to the remaining polar component of the field, namely the amplitude which describes the spatio-temporal damping/growth of the field. While the spatio-temporal gradients of the phase gives the real part of the wave 4-vector, the spatio-temporal gradients of the logarithmic wave amplitude can be modelled as the imaginary part of the wave 4-vector. If we add on the appropriate imaginary terms to the Taylor expansion of the phase above and then include these terms into the expression for the amplitude of the instantaneous local field, we would have

$A=A_{0} \exp (-\gamma t+\boldsymbol{q} \cdot \mathbf{x})$

with

$A_{0}:=A\left(t_{0}, \mathbf{x}_{0}\right) \exp \left(\gamma t_{0}-\boldsymbol{q} \cdot \mathbf{x}_{0}\right)$

where we have introduced the instantaneous local temporal amplitude modulation rate

$\gamma:=-\frac{\partial \ln A}{\partial t}$

and the instantaneous local spatial amplitude modulation vector

$\boldsymbol{q}=: \nabla \ln A$.

Notice that we have identified the imaginary part of the phase with the logarithm of the amplitude. $\gamma$ represents the instantaneous exponential rate of change in time of the wave amplitude, where $\gamma>0$ means a decrease in amplitude while $\gamma<0$ is an increase. Analogously, $|\boldsymbol{q}|$ is a measure of the local exponential variation in space of the wave amplitude while its direction is given by $\boldsymbol{q} /|\boldsymbol{q}|$. These parameters may indicate the existence of spatio-temporal damping/growth of the wave and even wave instabilities.

\footnotetext{
${ }^{1} \bmod (x, y)$ is the remainder of integer division $x / y$
} 
In the spectral domain, $\gamma$ and $\boldsymbol{q}$ have another interpretation: they are closely related to the instantaneous local bandwidth in frequency, $B_{f}$, and wave number, $B_{\kappa}$, respectively. The instantaneous bandwidth is defined for frequency as

$B_{f}:=\frac{1}{2 \pi}\left|\frac{1}{A(t, \mathbf{x})} \frac{\partial A(t, \mathbf{x})}{\partial t}\right|$

and for wave number

$B_{\kappa}:=\left|\frac{1}{A(t, \mathbf{x})} \nabla A(t, \mathbf{x})\right|$.

If $B_{\kappa} \ll \kappa$, then the bandwidth of the instantaneous local wavelength is approximately

$B_{\lambda} \approx 2 \pi \frac{B_{\kappa}}{|\kappa|^{2}}$.

For a detailed discussion of the instantaneous frequency bandwidth see Cohen (1995). For estimates of these quantities based on actual discretely measured data we use the same techniques for discretised spatio-temporal derivation as discussed in Sect. 3.

One important point with these bandwidths is that they provide a test of the geometrical optics approximation. We recall that this approximation assumes that the wave amplitude varies slowly in space over a wavelength and in time over wave period. Quantitatively, this means that we should have

$$
|\gamma| \ll|w| \quad \text { and } \quad|\boldsymbol{q}| \ll|\boldsymbol{\kappa}| .
$$

Thus, this provides the instantaneous local wave analysis, a simple condition to check for self-consistency.

Finally, we recall that the ratio of $w$ and $|\kappa|$ is the phase velocity the wave. Analogously, the ratio of $\gamma$ and $\boldsymbol{q}$ represents a velocity. The velocity can under certain conditions be the wave's group velocity. We will, however, not pursue this concept further in the current paper.

\section{The instantaneous local wave analysis algorithm}

We have presented theory and numerical estimates for an analysis which we will call instantaneous local wave (ILW) analysis. Starting from 4 time series of spacecraft data with known trajectories, the complex ILW is calculated as follows:

1. remove any unwanted signals, for instance, using bandpass filters;

2. re-interpolate the time series from each sensor to one common time sequence for all samples;

3. create analytical signals, based on the Hilbert transform, for each sensor signal by using, for example, Eq. (10)

4. calculate amplitude and phase of the analytical signal using the standard formulae (2) and (3);
5. find the spatio-temporal gradient components of phase using the discrete estimators (11) and (12);

6. find the bandwidths by using Eqs. (13) and (14).

The end result is the real 4-vector, $(w, \boldsymbol{\kappa})$ with corresponding bandwidths specified at each of the 4 spacecraft and for every sample in the time series.

The formulae developed here are explicitly for 4 spatially separated sensors, which is the minimal number for the full determination of $(\boldsymbol{w}, \boldsymbol{\kappa})$, but they can easily be extended to the case of more than 4 sensors.

The accuracy of the ILW estimates is a complicated issue. It depends on the relative temporal and spatial resolution between all the sensors and an estimate of the signal-to-noise ratio for the studied event. We postpone the error analysis of the ILW estimators to a future paper. Instead, in the following examples it is sufficient and illustrative to use the instantaneous bandwidth $B$ as an indication of the accuracy of some of the estimates.

\section{Examples}

We now apply ILW analysis to data taken from the EFW instrument (Gustafsson et al., 1997, 2001) on board the Cluster II space mission. EFW measures low frequency electric fields and the electric potential, the latter is a proxy for plasma density, from 4 spacecraft. We will look at two different types of events: a quasi-monochromatic wave pulse observed in the solar wind, in which we mainly use $(w, \boldsymbol{\kappa})$, and a dynamic current sheet structure observed in the Earth's magnetotail, in which $(\gamma, \boldsymbol{q})$ is used.

\subsection{Quasi-monochromatic wave pulse event}

Glassmeier et al. (2001) used an event on Cluster consisting of a quasi-monochromatic wave to illustrate the wave telescope technique. The wave telescope technique provides estimates of real wave vectors, therefore this event is appropriate for comparison with the ILW analysis.

The GSE Y-component of the electric field measured by the EFW instrument after post-processing with a $10-23 \mathrm{mHz}$ bandpass filter, is shown in Fig. 2a for Cluster spacecraft 1 during an approximately 10-min interval starting from 1 , January 2001 02:00:00 UT. The data exhibits a clear quasiperiodic wave which is slowly modulated in amplitude, as evidenced in the wave envelope (dashed red curve). For clarity, only the data from spacecraft 1 is shown in Fig. 2a but the other spacecraft measure a similar wave structure.

It is of course the differences between the spacecraft measurements which are the basis of the instantaneous local analysis of the wave. So, for example, in Fig. $2 b$ the temporal derivative of the phase at each spacecraft, that is the instantaneous local frequency $w(t, \mathrm{~s} / \mathrm{c})$, is shown. Figure $2 \mathrm{~b}$ illustrates the distinguishing features of $w(t, \mathrm{~s} / \mathrm{c})$ as opposed to ordinary frequency: each spacecraft sees a locally defined frequency for each temporal sample of $E_{y}$, compared to the 


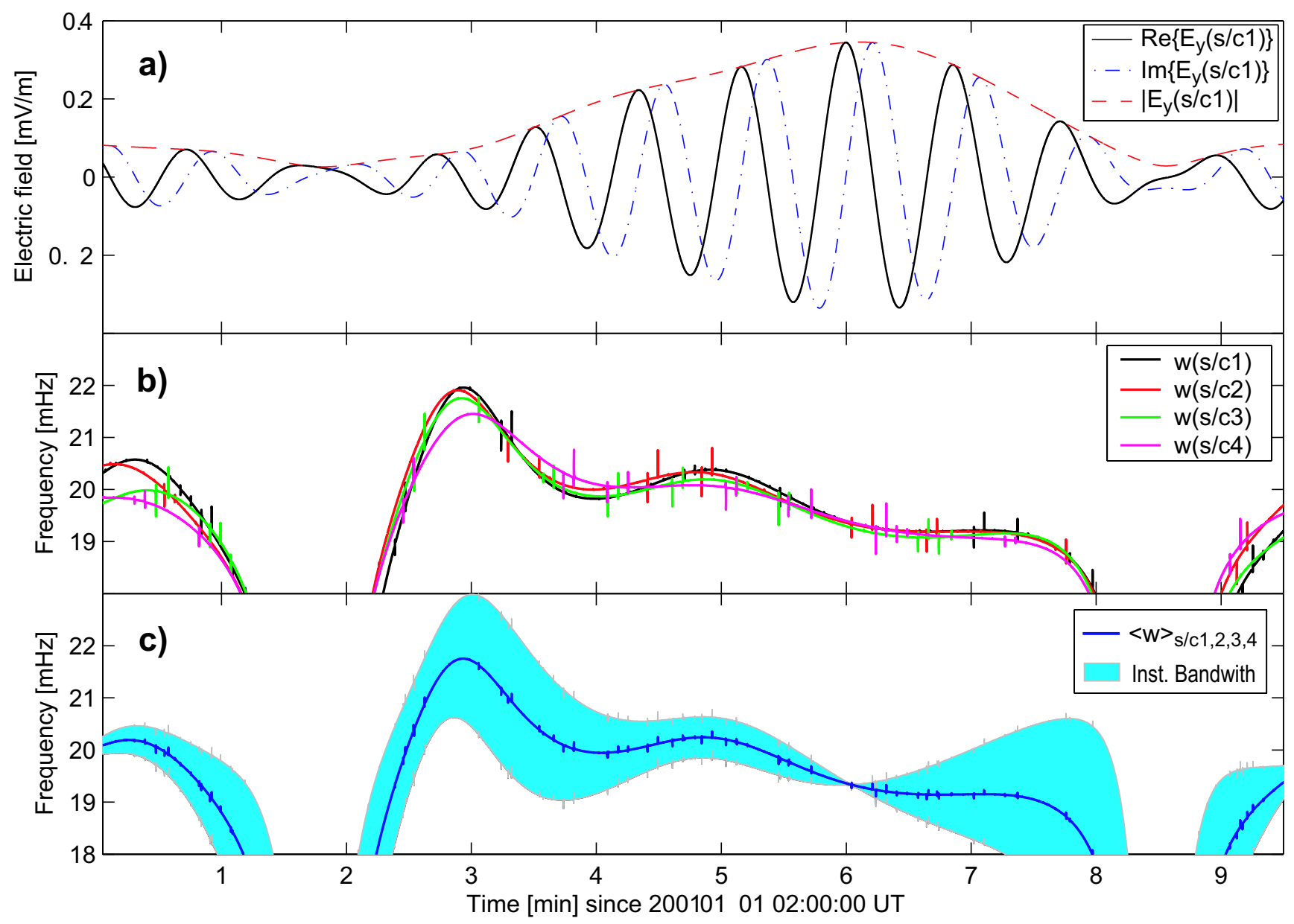

Fig. 2. A quasi-monochromatic wave observed over $10 \mathrm{~min}$ in the solar wind analysed using the instantaneous local wave analysis technique. The analysis starts from a real measured quantity, in this case the GSE Y-component of the electric field, $\operatorname{Re}\left\{E_{y}\right\}$, shown as a solid black curve in (a). The measured field is then Hilbert transformed to create the quadrature component $\operatorname{Im}\left\{E_{y}\right\}$ shown as a dot-dashed blue curve in (a). Taken together $\left(\operatorname{Re}\left\{E_{y}\right\}, \operatorname{Im}\left\{E_{y}\right\}\right)=E_{y}$, that is they make up the complex analytical signal of the electric field component and from which one calculates the envelope (amplitude) shown as a dashed red curve in (a). The phases are also calculated and their rate of change is the instantaneous local frequency $w(t, \mathrm{~s} / \mathrm{c})$ shown as the four (one colour per spacecraft) overlayed curves in (b). The average frequency for the Cluster quadruple, $\langle w\rangle$, is shown as a dark blue curve in (c). The analysis also provides an instantaneous bandwidth for the frequency estimate shown as a light shading region around the centre frequency curve in c). The bandwidth is useful for establishing the accuracy of $\langle w\rangle$. Among other things, the analysis reveals that the wave exhibits a weak, but detectable, modulation in frequency around $20 \mathrm{mHz}$.

ordinary notion of frequency which is only defined for the event as a whole. In Fig. $2 \mathrm{~b}$ the $w$ at each spacecraft are similar but not identical. The short spikes on the $w$ curves are an instrumental-numerical artefact and are not real.

For comparison with other techniques one can also calculate the frequency averaged over the 4 spacecraft, i.e. $\langle w\rangle=\sum_{\alpha=1}^{4} w^{(\alpha)}(\sigma)$, shown as a dark blue curve in Fig. 2c. A more refined frequency could be obtained by interpolating the 4 frequencies to the barycentre of the Cluster tetrahedron, see Paschmann and Daly (1998). This single barycentric frequency could be combined with the single estimate of the local wave vector $\boldsymbol{\kappa}$ to form a single wave 4-vector for the wave as seen by the spacecraft tetrahedron.

An indication of the accuracy of the average frequency is found from the spacecraft averaged instantaneous bandwidth which is plotted as a light blue area centred on $\langle w\rangle$.
The direction of the instantaneous local wave vector for the event is shown in Fig. 3. It is rather steady and centred on a direction slightly below the anti-sunward direction. The wave length of this wave, derived as the average of $2 \pi /|\kappa|$ over the main pulse, is $12024 \pm 1800 \mathrm{~km}$, where the spread is based on the measured $B_{\kappa}$ given by Eq. (14). The phase velocity of the wave, computed from Eq. (8) using the electric field data presented here, was found to be around $240 \mathrm{~km} / \mathrm{s}$. Our results are comparable to Glassmeier (2003), who used magnetic field data and found the propagation direction to be roughly anti-sunwards and a wave length of $12571 \mathrm{~km}$.

Our analysis of the event reveals that, although the frequency of the wave is almost constant, there is a small but detectable modulation of the frequency around the $20 \mathrm{mHz}$ average. A similar modulation is seen in the change of the wave vector direction in GSE X-Y plane in Fig. 3. 


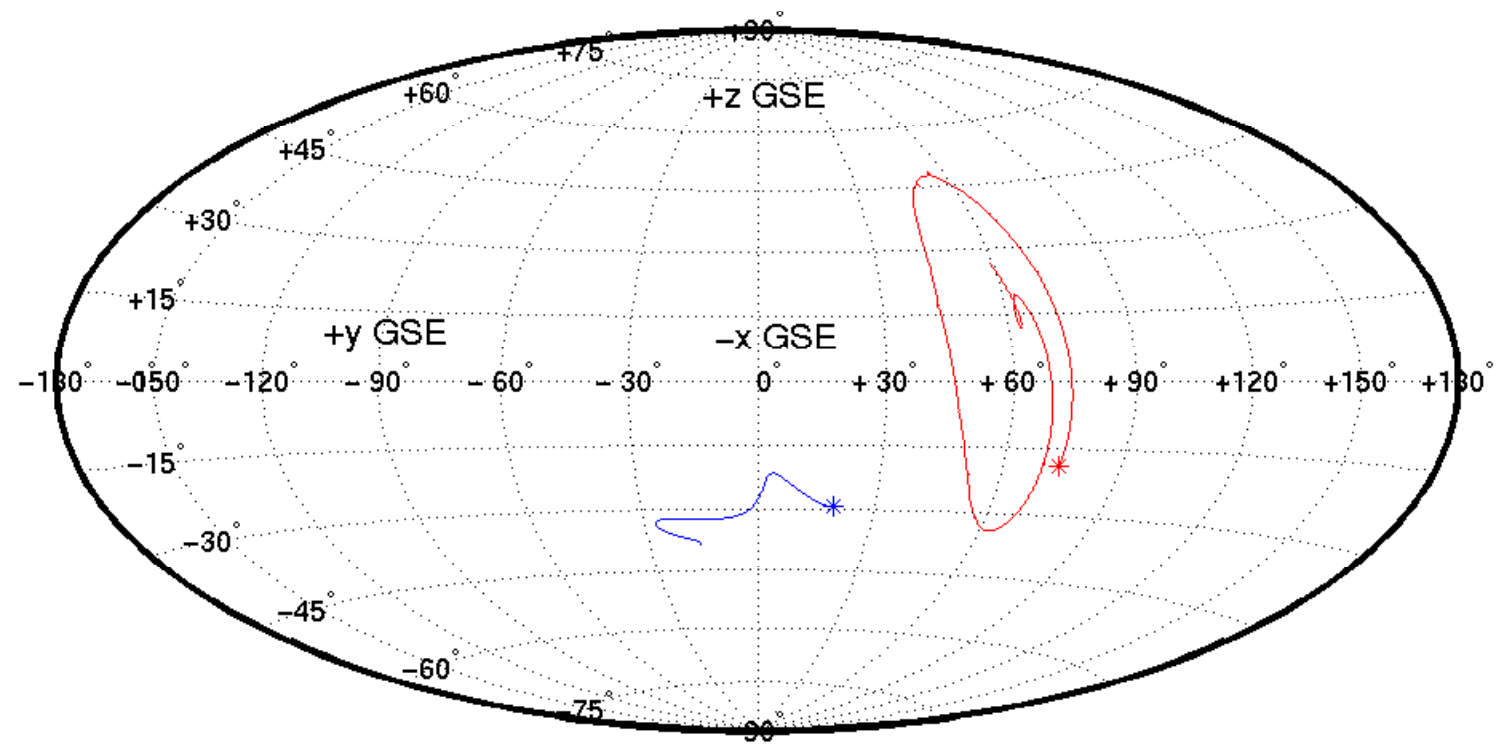

Fig. 3. Polar map of the instantaneous local wave vector direction of the wave packet shown in Fig. 2. The blue (red) curve shows the real (imaginary) local wave vector direction in GSE coordinates (looking towards the Earth at the origin and with the Sun at $\pm 180^{\circ}$ on the horizontal parallel). The star on one end of the curve mark the beginning of the main wave event at 1 January 2001, 01:59:30 UT and the curves continue until the end of the main event which is $300 \mathrm{~s}$ after the start. The wave is estimated to be propagating mainly along the anti-sunward direction (-X GSE) at a velocity of $240 \mathrm{~km} / \mathrm{s}$, consistent with a low frequency wave convected along the solar wind. The direction of the imaginary part of the wave vector is mainly orthogonal to the real part.

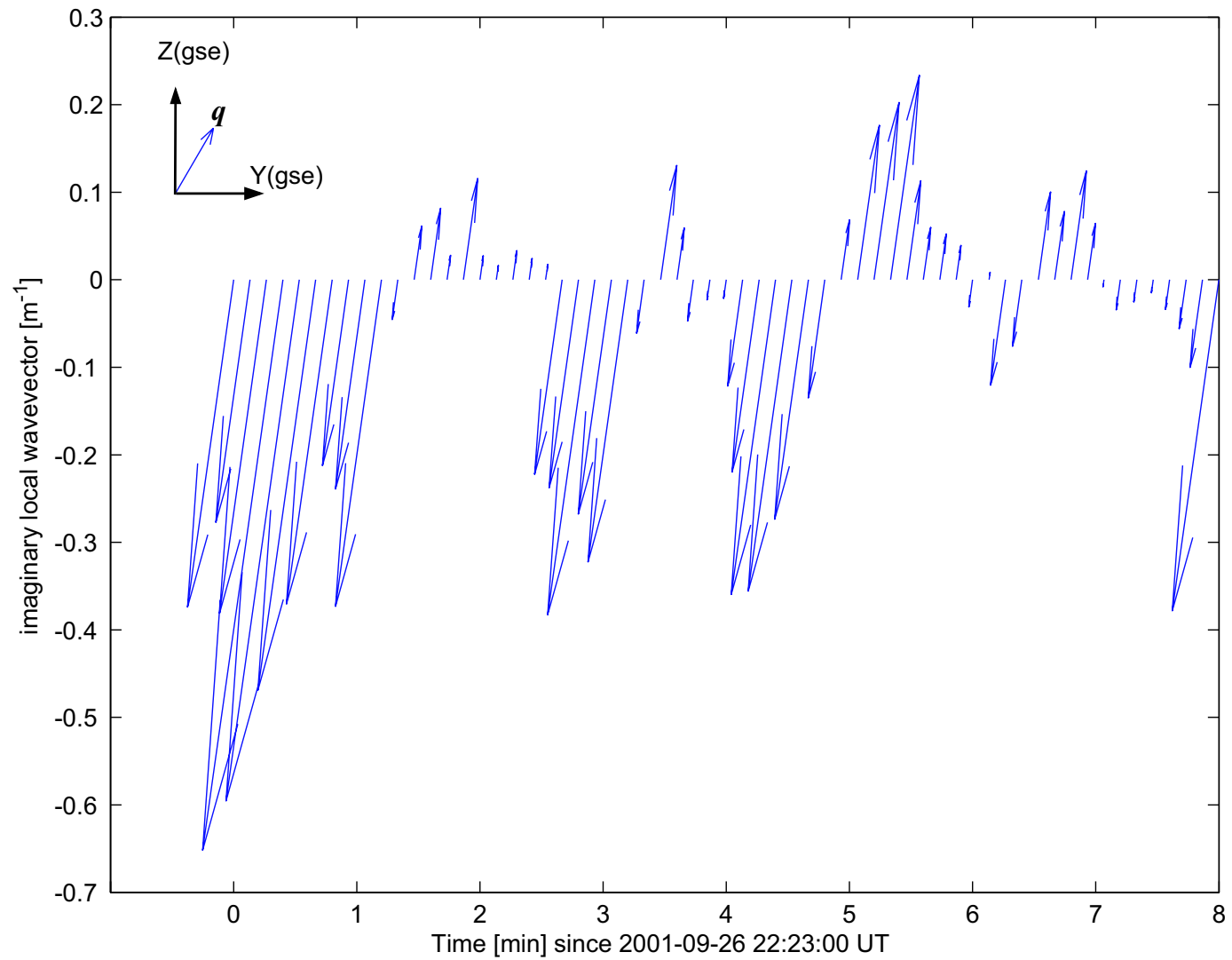

Fig. 4. Local spatial amplitude modulation rate vector in the GSE Y-Z plane during the current sheet flapping event. Oscillations of the current sheet on minute time scales is visible. 
A possible cause of this effect is that the wave source is wobbling, thereby producing undulating Doppler shifts in the emission frequency; although other temporal changes in the source or the intervening medium cannot be excluded.

Note that although the wave discussed here was quasimonochromatic, the ILW analysis is by no means limited to such cases. Other types of waves encountered in space physics for which ILW analysis would be particularly useful are whistlers and solitons. The former is characterised by its changing frequency and latter by its wide coherent spectrum. An alternative technique for deriving instantaneous frequency appropriate for chorus elements was given by Santolík et al. (2003).

\subsection{Dynamic boundary transition event}

The ILW analysis is not limited to oscillating waves as in the last example. Recently, Kohlmann (1996) used the HT to detect edges in 2-D images. There are numerous examples in space physics of edge-like structures. One can analyse such plasma structures with ILW, but in contrast to the previous wave-like example, one uses mainly the imaginary part of $\boldsymbol{\kappa}$. This is because the imaginary part of $\boldsymbol{\kappa}$ describes spatial damping/growth which is a measure of spatial structuring. Physically, this type of analysis is useful when the data represents transitions between two regions, that is, boundary or surface crossing. We now look at such a case.

On 26 September 2001, Cluster passed in and out of the current sheet (CS) in the Earth's magnetotail due to a flapping motion of the CS. We have examined the same event using ILW analysis of the electric potential from EFW as a proxy for the plasma density. In Fig. 4, we show the GSE Y-Z plane of the instantaneous local amplitude modulation vector $\boldsymbol{q}$ given in Eq. (5). As can be seen there is an approximately sinusoidal oscillation of the vectors, mainly in the GSE Z direction. There is a noticeable asymmetry between the downward and upward phase of the oscillation. This oscillation matches well with that found by Sergeev et al. (2003), who used minimum variance analysis (MVA) and inter-spacecraft timing.

\section{Conclusions and comparison with other techniques}

In this paper we have presented an analysis method for spatio-temporal aspects of fields from multipoint measurements which we called the instantaneous local wave (ILW) analysis.

Some features of the ILW analysis are

- does not assume stationary or homogeneity;

- can be used on waves, boundaries, or structures;

- estimates wave characteristics instantaneously;

- useful for tracking gradual changes of wave properties;

- provides not only frequency but also the damping rate of waves;
- does not require knowledge of wave polarisation;

- provides a concise representation of single-component waves.

The drawbacks of the ILW analysis, on the other hand, are that it does not allow for multicomponent waves, (that is, when several waves or structures coalesce in space-time) and that it requires a high signal-to-noise ratio. In the multicomponent wave it is possible, however, in many cases to apply some filtering process to extract the signal of interested before using the ILW analysis. This can be done by using classical filter theory or purpose built methods, such as the empirical mode decomposition method proposed by Huang et al. (1998). We have also developed an extended ILW analysis to handle the multicomponent wave which will be featured in a sequel paper. This extended method will also allow for the presence of noise in the signal.

Besides finding that the analysis produces results consistent with other techniques, the use of the ILW analysis also produced new findings and it could be applied to cases where other methods cannot, such as the continuous quantification of current sheet flapping or rapid dynamical changes in observed wave packets. We conclude therefore that ILW could become a useful tool for multipoint spatio-temporal studies in space physics, such as in conjunction with multipoint raytracing (Parrot et al., 2003).

Acknowledgements. This work was sponsored by PPARC ref: PPA/G/S/1999/00466 and PPA/G/S/2000/00058. The authors wish to thank M. André and A. Vaivads for their help with the use of EFW data.

Topical Editor T. Pulkkinen thanks O. Santolík and another referee for their help in evaluating this paper.

\section{References}

Balikhin, M. A., Pokhotelov, O. A., Walker, S. N., Amata, E., André, M., and Dunlop, M.: Minimum variance free wave identification: Application to Cluster electric field data in the magnetosheath, Geophys. Rev. Lett., 30, 1508, 2003.

Beall, J. M., Kim, Y. C., and Powers, E. J.: Estimation of wavenumber and frequency spectra using fixed probe pairs, J. Appl. Phys., 53, 3933-3940, 1982.

Boashash, B.: Time-frequency signal analysis: Methods and Applications, Longman Cheshire, 1992.

Born, M. and Wolf, E.: Principles of Optics, Cambridge University Press, Cambridge, 7th Ed., 1999.

Bülow, T. and Sommer, G.: Hypercomplex Signals - A Novel Extension of the Analytic Signal to the Multidimensional Case, IEEE Transactions on signal processing, 49, 2844-52, 2001.

Cohen, L.: Time-frequency Analysis: Theory and Applications, Prentice Hall PTR, 1995.

Glassmeier, K.-H.: Correction to "Cluster as a wave telescope - first results from the fluxgate magnetometer", K. H. Glassmeier et al., Ann. Geophys., 21, 1071-1072, 2003.

Glassmeier, K.-H., Motschmann, U., Dunlop, M., Balogh, A., Acuña, M. H., Carr, C., Musmann, G., Fornaçon, K.-H., Schweda, K., Vogt, J., Georgescu, E., and Buchert, S.: Cluster 
as a wave telescope - first results from the fluxgate magnetometer, Ann. Geophys., 19, 1439-1447, 2001.

Gustafsson, G., Boström, R., Holback, B., Holmgren, G., Lundgren, A., Stasiewicz, K., and Åhlén, L., Mozer, F. S., Pankow, D., Harvey, P., Berg, P., Ulrich, R., Pedersen, A., Schmidt, R., Butler, A., Fransen, A. W. C., Klinge, D., Thomsen, M., Fälthammar, Lindqvist, P.-A., Christenson, S., Holtet, J., Lybekk, B., Sten, T. A., Tanskanen, P., Lappalainen, K., and Wygant, J. : The electric field and wave experiment for the Cluster mission, Space Sci. Rev., 79, 137, 1997.

Gustafsson, G., André, M., Carozzi, T., Eriksson, A. I., Fälthammar, C.-G., Grard, R., Holmgren, G., Holtet, J. A., Ivchenko, N., Karlsson, T., Khotyaintsev, Y., Klimov, S., Laakso, H., Lindqvist, P.-A., Lybekk, B., Marklund, G., Mozer, F., Mursula, K., Pedersen, A., Ppielawska, B., Savin, S., Stasievicz, K., Tanskanen, P., Vaivads, A., and Wahlund, J.-E.: First results of electric field and density observations by Cluster EFW based on initial months of operation, Ann. Geophys., 19, 1219-1240, 2001.

Hahn, S. L.: Hilbert Transforms in Signal Processing, Artech House, 1997.

Huang, N. E., Shen, Z., Long, S. R. et al.: The empirical mode decomposition and the Hilbert spectrum for nonlinear and nonstationary time series analysis, Proc. R. Soc. Lond. A, 454, 903995, 1998

Kintner, P. M., LaBelle, J., Kelley, M. C., Cahill Jr., L. J., Moore, T., and Arnoldy, R.: Interferometric phase velocity measurement, Geophys. Rev. Lett., 11, 19-22, 1984.

Kohlmann, K.: Corner detection in natural images based on the 2-D Hilbert transform, Signal Processing, 48, 225-234, 1996.
Parrot, M., Santolík, O., Cornilleau-Wehrlin, N., Maksimovic, M., and Harvey, C.: Magnetospherically reflected chorus waves revealed by ray tracing with Cluster data, Ann. Geophys., 21, 1111-1120, 2003.

Paschmann, G. and Daly, P.: Analysis methods for Multi-Spacecraft Data, Scientific report SR-001, ESA publications division, 1998.

Perrin, F., Zhu, Y. M., and Goutte, R.: Extension of the notion of analytic signal for multidimensional signals, Application to images, in: Signal Processing III: Theories and applications, proceedings of EUSIPCO-86 third European Signal Processing Conference, edited by Young, I. T., EURASIP, Elsevier Science publishers B.V. (North-Holland), 677-680, 1986.

Pinçon, J. L. and Motschmann, U.: Magnetospherically reflected chorus waves revealed by ray tracing with Cluster data, in Analysis methods for Multi-Spacecraft Data, edited by Paschmann, G. and Daly, P., ESA, 1998.

Santolík, O., Gurnett, D. A., Pickett, J. S., Parrot, M., and Cornilleau-Wehrlin, N.: Spatio-temporal structure of storm-time chorus, J. Geophys. Res., 108, 2003.

Sergeev, V., Runov, A., Baumjohann, W., Nakamura, R., Zhang, T. L., Volwerk, M., Balogh, A., Réme, H., and Sauvaud, J. A.: Current sheet flapping motion and structure, Geophys. Rev. Lett., 30, 1327, 2003.

Storey, L. R. O. and Lefeuvre, F.: Theory for the Interpretations of Measurements of the Six components of a Random Electromagnetic Wave Field in Space, Space Research, 14, 381-386, 1974.

Whitham, G. B.: Linear and Nonlinear Waves, John Wiley \& Sons, 1974. 